\title{
La conservation du poisson au Sénégal : utilisation d'une souche locale de Lactococcus lactis
}

\author{
Michel Bakar Diop 1 \\ Jacqueline Destain ${ }^{1}$ \\ Philippe Thonart ${ }^{1}$ \\ Robin Dubois-Dauphin ${ }^{1}$ \\ Emmanuel Tine ${ }^{2}$ \\ ${ }^{1}$ Centre wallon de biologie industrielle \\ (CWBI)/Gembloux Agricultural University \\ 2, passage des Déportés \\ B-5030 Gembloux \\ Belgique \\ <diopmb@yahoo.fr> \\ 2 Université Cheikh Anta Diop (Ucad) \\ École supérieure polytechnique \\ BP 5085 \\ Dakar \\ Sénégal \\ <emmanueltine@yahoo.fr>
}

\begin{abstract}
Résumé
L'effet antimicrobien du surnageant de culture neutralisé (SCN) bactéricide issu de Lactococcus lactis CWBI-B1410 d'origine locale, utilisé seul ou en combinaison avec du chlorure de sodium ( $\mathrm{NaCl}$ ) comme conservateurs, a été évalué sur poissons maigre (Pomadasys jubelini), moyennement gras (Polydactylus quadrifilis) et gras (Arius beudeloti)] au Sénégal. Les poissons ont été achetés dans un marché local, éviscérés, lavés avec de l'eau de robinet potable, puis filetés. Cent millilitres de SCN de CWBI-B1410 non salé et salé ont été additionnés dans 100 grammes de filets (concentration finale de $\mathrm{NaCl}$ entre 0 et $7 \%$ ) dans des bocaux en verre conservés à $10^{\circ} \mathrm{C}$. L'évolution de la flore mésophile totale (FMT) des filets a été comparée à celles de filets traités avec du SCN non salé et salé (concentration finale de $\mathrm{NaCl}$ entre 0 et $7 \%$ ), issu de L. lactis LMG6890 ne produisant pas de bactériocine. Un niveau de FMT de $10^{6}$ micro-organismes par gramme (ufc/g) a été considéré comme la fin de la durée de conservation. Le niveau de la flore mésophile totale des filets crus atteignait 5,74 log ufc/g. L'ajout de SCN de CWBI-B1410 dans les filets de poisson maigre réduit la flore mésophile totale de $1 \log$ ufc/g et stabilise la flore pendant 4 jours - correspondant à la durée de conservation de ces filets à $10{ }^{\circ} \mathrm{C}$, contre 0,5 jour pour le contrôle négatif. L'addition de SCN de CWBIB1410 salé sur les poissons ( $\mathrm{NaCl}, 7 \%$ ) réduit davantage la FMT et retarde sa croissance à $10^{\circ} \mathrm{C}$, entraînant comme résultat l'augmentation de la durée de conservation de respectivement $12,7,5$ et 8 jours pour le poisson maigre, moyennement gras et gras. Ces résultats suggèrent que cette stratégie peut constituer un moyen convenable d'améliorer la conservation des produits halieutiques tropicaux.
\end{abstract}

Mots clés : antimicrobien ; bactéricide ; conservation (stockage) ; filet de poisson ; sel ; Sénégal.

Thèmes : technologie agro-alimentaire ; technologie, récolte, transport.

\section{Abstract \\ Use of Lactococcus lactis CWBI-B1410 screened from local food products for conserving fish in Senegal}

The antimicrobial effect of the bactericidal neutralized culture supernatant (NCS) from Lactococcus lactis CWBI-B1410 screened from local food products and used as a preservative alone or in combination with sodium chloride was evaluated on lean (Pomadasys jubelini), moderately fat (Polydactylus quadrifilis), and fat (Arius heudeloti) fish in Senegal. Fish samples were purchased from a local market, eviscerated, cleaned using drinkable water and filleted. One hundred milliliters each of non-salted and salted CWBIB1410 neutralized culture supernatant were added to $100 \mathrm{~g}$ of fish fillets ( $\mathrm{NaCl}$ final concentration from 0 to $7 \%$ ) in separate glass jars which were stored at $10^{\circ} \mathrm{C}$. The evolution of the microbial flora [total aerobic mesophilic flora (TMF)] of these fillets was compared to those of fillets treated in using the non-salted and salted ( $\mathrm{NaCl}$ final concentration from 0 to 7\%) NCS from the non-producing bacteriocin L. lactis LMG6890 strain. A $10^{6} \mathrm{cfu} / \mathrm{g}$ TMF level was considered as the end of shelf-life. The microbial flora of raw fish fillets reached a level of $5.74 \mathrm{log} \mathrm{cfu} / \mathrm{g}$. The addition of CWBI-B1410 NCS to the lean fish fillets lowered the TMF counts of $1 \mathrm{log} \mathrm{cfu} / \mathrm{g}$ and stabilized the flora for four days, corresponding to the shelf-life of the fillets, against 0.5 days for those treated with LMG6890 NCS. The addition of salted CWBI-B1410 NCS to fish (at NaCl final concentration of $7 \%$ ) enhanced the decrease in TMF of fish and delayed the increase of bacteria counts at $10^{\circ} \mathrm{C}$, resulting in an extension of shelf-life of $12,7.5$ and 8 days for lean, 
moderately fat, and fat fish, respectively. These data suggest that this strategy can be a suitable means for improving bacterial quality and preservation of tropical fish commodities.

Key words: antimicrobials; bactericides; conservation (storage); fish fillets; salt; Senegal.

Subjects: agrifood technologies; technology, crop, transport.

e poisson constitue une source de protéines sensiblement identique à celle de la viande (Guiraud, 1998 ; Stansby, 1962) et la principale source de protéines d'origine animale des populations dans de nombreux pays en développement. Il est toutefois une denrée éminemment périssable. Sa dégradation est en effet la résultante de l'activité in situ des enzymes et des micro-organismes, notamment des bactéries (Baird-Parker, 2000 ; Gram et Dalgaard, 2002). Or, certains de ces micro-organismes tels que les entérobactéries qui produisent notamment des amines biogéniques, sont la cause de maladies chez l'homme (Olsen et al., 2000).

Les méthodes modernes de conservation du poisson basées sur l'utilisation du froid (réfrigération et congélation) fournissent des produits d'excellente qualité (Boyd et al., 1992). Malheureusement, il s'agit de méthodes industrielles, supposant l'existence d'une infrastructure lourde, coûteuse et dévoreuse d'énergie. Elles sont très peu répandues dans les pays du Sud où la conservation par salage demeure prédominante (Gérard, 1989, Fellows, 1997). Certains travaux ont montré que l'utilisation du sel en combinaison avec des agents antimicrobiens d'origine bactérienne tels que les bactériocines, améliore la stabilité et la qualité microbiologique des produits de pêche conservés à 4-8 ${ }^{\circ} \mathrm{C}$ (Degnan et al., 1994 ; Wessels et Huss, 1996; Ghalfi et al., 2006 ; Einarsson et al., 1995).

Les bactériocines sont des substances peptidiques (ou protéines) produites par certaines bactéries et qui ont un effet inhibiteur sur d'autres bactéries (De Vuyst et Vandamme, 1994). Au cours d'une sélection, à partir de produits alimentaires transformés artisanalement, de bactéries lactiques d'origine sénégalaise productrices de bactériocine, la souche Lactococcus lactis CWBI-B1410, qui porte le gène codant pour la nisine $\mathrm{A}$, a été sélectionnée, du fait de son activité bactéricide importante dans le surnageant de culture neutralisé (SCN) (Diop et al., 2006 ; Diop et al., 2007).

L'objectif de cette étude est d'évaluer l'effet antimicrobien du SCN issu de L. lactis CWBI-B1410, utilisé seul et en combinaison avec du chlorure de sodium comme conservateur sur différents poissons, maigre (sompat [Podamasys jubelini]), moyennement gras (capitaine [Polydactylus quadrifilis]) et gras (mâchoiron [Arius beudeloti]) - ces trois espèces de poisson étant prédominantes dans les débarquements de la pêche artisanale au Sénégal (DOPM, 2004).

La contamination microbienne des produits halieutiques destinés à l'alimentation humaine est exprimée en nombre de micro-organismes par gramme (ufc/g) en ce qui concerne la flore mésophile totale (FMT). Pour les produits frais ou congelés, ronds ou en filets, la limite de contamination microbienne généralement acceptable est définie à $<10^{5}$ ou $5 \times 10^{5} / \mathrm{g}$ (Guiraud, 1998). Par conséquent, un niveau de flore totale de $10^{6} \mathrm{ufc} / \mathrm{g}$ a été considéré comme la fin de la durée de conservation des filets de poissons au cours des essais de préservation à $10^{\circ} \mathrm{C}$.

\section{Matériel et méthode}

\section{Souches lactiques, milieux et conditions de cultures}

L. lactis CWBI-B1410, qui présente une activité antibactérienne de type bactéricide et le gène codant pour la nisine $\mathrm{A}$, a été sélectionnée à partir de bactéries lactiques isolées à partir de produits alimentaires artisanaux d'origine sénégalaise (Diop et al., 2006 ; Diop et al., 2007). L. lactis subsp. lactis LMG6890, qui n'a pas de capacité à produire une bactériocine, a été obtenue à partir de la collection du Laboratoire de microbiologie de l'université de Gand (LMG) (Belgique). Cette souche est utilisée comme contrôle négatif au cours des essais de conservation des filets de poisson. Pediococcus pentosaseus, issu de la collection du Centre wallon de biologie industrielle (CWBI) de l'université de Gembloux (FUSAGx), est utilisé comme souche indicatrice pour évaluer l'activité antibactérienne in vitro et in situ (sur filet de poisson) des SCN des souches testées, éventuellement additionnés de chlorure de sodium.

Les deux souches CWBI-B1410 et LMG6890, conservées à $-80{ }^{\circ} \mathrm{C}$ sur billes, ont été repiquées sur gélose de Man, Rogosa, Sharpe (MRS). Deux colonies de chacune des souches ont été inoculées dans $10 \mathrm{~mL}$ de bouillon MRS et incubés pendant 12 heures à $30^{\circ} \mathrm{C}$. Cinq millilitres de ces précultures ont été inoculés dans $500 \mathrm{~mL}$ de bouillon MRS, incubés pendant 12 heures à $30^{\circ} \mathrm{C}$. Les cultures ont été centrifugées (Sigma 2-4, Germany) à 2282 x g pendant 20 minutes. Le potentiel hydrogène $(\mathrm{pH})$ des surnageants de culture des deux souches a été déterminé par mesure (Eutech instrument, 219 208, Singapour), puis neutralisé à $\mathrm{pH} 6$ par ajout (1\%, v/v) d'une solution de $\mathrm{NaOH} 5 \mathrm{~N}$.

\section{Activité antimicrobienne des SCN des deux souches lactiques}

L'activité antibactérienne sur les SCN issus de CWBI-B1410 et LMG6890 a été déterminée par adaptation de la technique de dilution critique décrite par Barefoot et Klahenammer (1983), en utilisant la souche $P$. pentosaseus comme souche indicatrice. Cent dix microlitres d'une culture fraîche (16 heures à $37^{\circ} \mathrm{C}$ sur $10 \mathrm{~mL}$ de bouillon MRS) de $P$. pentosaseus ont été déposés au fond d'une boîte de Petri stérile, puis suspendus dans $20 \mathrm{~mL}$ de gélose MRS en surfusion à $50^{\circ} \mathrm{C}$. Les boîtes ont été incubées pendant 40 minutes. à $4{ }^{\circ} \mathrm{C}$, pour que l'agar se solidifie rapidement, et des puits de $0,65 \mathrm{~mm}$ de diamètre ont été 
creusés dans la gélose à l'aide d'une pipette Pasteur stérile sous hotte stérile.

Deux cents microlitres de SCN pasteurisé (10 minutes, $80^{\circ} \mathrm{C}$ ) ont été dilués successivement de $1 / 2$ dans du tampon phosphate $50 \mathrm{mM}$ (millimolaires) $\mathrm{pH}$ 6. Cinquante microlitres de chaque dilution ont été déposés dans les puits. Les boîtes ont été séchées pendant 40 minutes sous hotte (à demi ouverte), pour laisser les composés contenus dans les solutions diffuser de manière radiale dans la gélose, puis incubées à $37^{\circ} \mathrm{C}$ pendant une nuit, pour détecter la présence d'une d'inhibition de croissance. Celle-ci se manifeste par la présence d'une zone claire tout autour du puits. L'activité antibactérienne du SCN exprimée en Unités arbitraires $(\mathrm{AU} / \mathrm{ml})$ correspond à l'inverse de la dernière dilution montrant une zone d'inhibition.

L'activité inhibitrice a été également mesurée sur les jus de poissons au cours du temps, par la méthode décrite cidessus. Cette activité résiduelle a été exprimée en \%, en considérant celle sur le SCN issu de la souche CWBI-B1410 comme étant de $100 \%$.

\section{Origine des poissons, conditions de filetage, et teneur en lipides totaux des filets}

Les poissons ont été achetés au marché artisanal de Soumbédioune (Dakar Fann, Sénégal) durant toute la durée de l'étude, du 10 juillet 2006 au 4 octobre 2006 . Les poissons ont une longueur et un poids variant respectivement de 40 à 50 centimètres et de 700 à 900 grammes. Ils ont été éviscérés et écaillés sur place par les "femmes nettoyeuses" (DieiOuadi, 2005), puis transportés rapidement au Laboratoire L-MAGI ESP à Dakar (au bout de 15 minutes) dans des sacs plastiques fermés hermétiquement. Ils ont été lavés avec de l'eau du robinet, potable, puis filetés stérilement. Deux filets de 200 grammes - munis de peau ont été retirés de chaque poisson, et chacun subdivisé en deux parties égales pour les essais de préservation.

La teneur en lipides totaux des filets des différents poissons a été estimée par la technique de Folch (1957). Les constituants lipidiques des aliments et les poissons gras sont en effet connus respectivement pour leurs propriétés à interagir avec les molécules de bactériocines (Aasen et al., 2003) et absorber plus lentement le sel (OSU Extension Service, 1993).

\section{Solutions de préservation et conditionnement des filets de poissons}

Les SCN de LMG6890 et de CWBI-B1410 ont été additionnés de $\mathrm{NaCl}(0,8,12$ et $14 \%)$. Le pH des solutions salées et non salées a été ajusté à 6. Les solutions ont été pasteurisées $\left(80{ }^{\circ} \mathrm{C}\right.$ pendant 10 minutes) puis refroidies à $10^{\circ} \mathrm{C}$ pendant 2 heures avant d'être utilisées pour imprégner les filets. Cent millilitres des différentes solutions ont été ajoutés dans 100 grammes de filets de poissons dans un bocal en verre stérile conservé à $10^{\circ} \mathrm{C}$. La concentration finale en $\mathrm{NaCl}$ dans les jus de poissons ainsi salés varie entre 0 et $7 \%$.

\section{Dénombrement de la microflore des filets de poissons}

La flore aérobie mésophile totale (FMT) a été dénombrée sur PCA (gélose pour dénombrement des micro-organismes aérobies totaux) additionné de $\mathrm{NaCl}$
(0,5\%). Le dénombrement a été réalisé toutes les 48 heures. Deux grammes de chair de poisson ont été suspendus dans $18 \mathrm{~mL}$ d'eau contenant de la peptone $(0,1 \%)$ et du $\mathrm{NaCl}(0,5 \%)$ stérile dans un tube Falcon de $50 \mathrm{~mL}$, et mélangé vigoureusement pendant 2 minutes à l'aide d'un vortex. La suspension a été ensuite diluée de $1 / 10$ successivement, et $100 \mu \mathrm{L}$ de chaque dilution ont été étalés en surface. Les boites ont été incubées à $30^{\circ} \mathrm{C}$ pendant 48 heures avant que les colonies ne soient comptées. Un niveau de FMT de $10^{6} \mathrm{ufc} / \mathrm{g}$ a été considéré comme la fin de la durée de conservation. Les résultats présentés dans cette étude sont la moyenne de deux essais séparés sur des échantillons différents pour chaque poisson.

\section{Résultats et discussion}

Le $\mathrm{pH}$ des surnageants de culture des deux souches a été déterminé à 4,3 $\pm 0,2$ (pour CWBI-B1410) et 4,4 $\pm 0,3$ (pour LMG6890). Après neutralisation du pH à 6, l'activité antibactérienne in vitro sur le SCN de CWBI-B1410 a été déterminée à

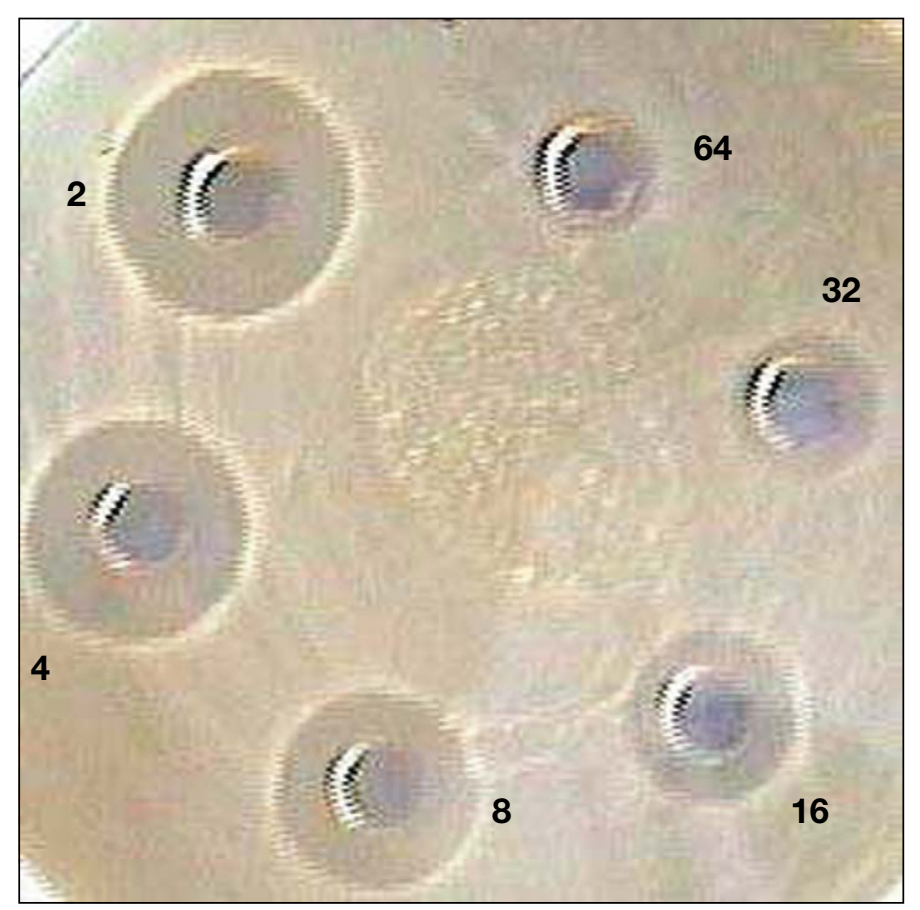

Figure 1. Activité antibactérienne du surnageant de culture neutralisé (SCN) de L. lactis subsp. lactis CWBI-B1410 contre la souche de $P$. pentosaseus.

Figure 1. Antibacterial activity of neutralized culture supernatant (NCS) of L. lactis subsp. lactis CWBIB1410 against $P$. pentosaseus.

Le SCN a été dilué de 1/2 (2) à 1/64 (64). La dernière dilution montrant une zone d'inhibition étant la dilution 64. 
$1280 \mathrm{AU} / \mathrm{mL}$, la dernière dilution montrant une zone d'inhibition étant la dilution 64 (figure 1). En revanche, aucune activité antibactérienne in vitro n'a été détectée sur le SCN issu de la souche LMG6890 (résultats non présentés).

Le niveau de la FMT des filets crus a été déterminé à 5,74 \pm 0,07 log base $10 \mathrm{ufc/g}$ pour le sompat, 5,37 $\pm 0,06 \mathrm{log}$ ufc/g pour le capitaine et 5,65 $\pm 0,29 \mathrm{log}$ ufc/g pour le mâchoiron (figure 2), alors que la limite d'acceptabilité est de $10^{6}$ ufc/g. Ce niveau de contamination microbienne est similaire à ceux publiés par Gram (1992).

La teneur en lipides totaux a été estimée à 2,8\% pour le sompat (poisson maigre), 3,28 \% pour le capitaine (poisson moyennement gras) et $17,75 \%$ pour le mâchoiron (poisson gras).

L'addition de SCN bactéricide de CWBIB1410 dans les filets du poisson maigre entraîne une réduction de la FMT de
$1 \mathrm{log}$ ufc/g alors qu'aucun effet antimicrobien n'a été observé contre la flore dans les filets traités avec le SCN de LMG6890. Cependant, la FMT, dans les filets additionnés de SCN de CWBI-B1410, entame une croissance (phénomène de rebond) au quatrième jour de conservation (figure 2A).

L'utilisation de SCN de CWBI-B1410 salés comme solution de préservation à $10{ }^{\circ} \mathrm{C}$ entraîne une réduction plus marquée $(1,5$ $\log \mathrm{ufc} / \mathrm{g}$ ) et une meilleure stabilisation de la FMT. Il en résulte des augmentations de durée de conservation des filets de 3, 7, 9 et 12 jours pour des concentrations finales en $\mathrm{NaCl}$ atteignant $0,4,6$ et $7 \%$ (figures $2 A, 2 B, 2 C$ et $2 D$ ). De même, des augmentations de durée de conservation de 7,5 et 8 jours ont été obtenues respectivement, pour les filets de capitaine et de mâchoiron (Podamassys jubelini) traités avec du SCN de CWBI-B1410 salé (concentration finale en $\mathrm{NaCl}$ de $7 \%$ ), (figure 3).

Des augmentations de 21 jours de la durée de conservation des crevettes salées additionnées de surnageant de culture bactéricide issu de L. lactis SIK83 ont été publiées par Einarsson et al. (1995). Les conditions de travail de ces auteurs diffèrent des nôtres par une température de conservation plus faible $\left(4{ }^{\circ} \mathrm{C}\right)$ et un niveau de contamination microbienne initiale plus faible (10 ${ }^{2-3}$ ufc/g). De même, Gram (1992) a publié une baisse de la microflore de poissons tropicaux de $10^{7} \mathrm{ufc} / \mathrm{g}$ à $10^{3-4} \mathrm{ufc} / \mathrm{g}$ et sa stabilisation pendant 7 jours par conditionnement par le froid (100 grammes de poisson dans 100 grammes de glace). Toutefois cette stratégie apparaît plus contraignante et coûteuse en énergie que celle décrite dans cette étude.

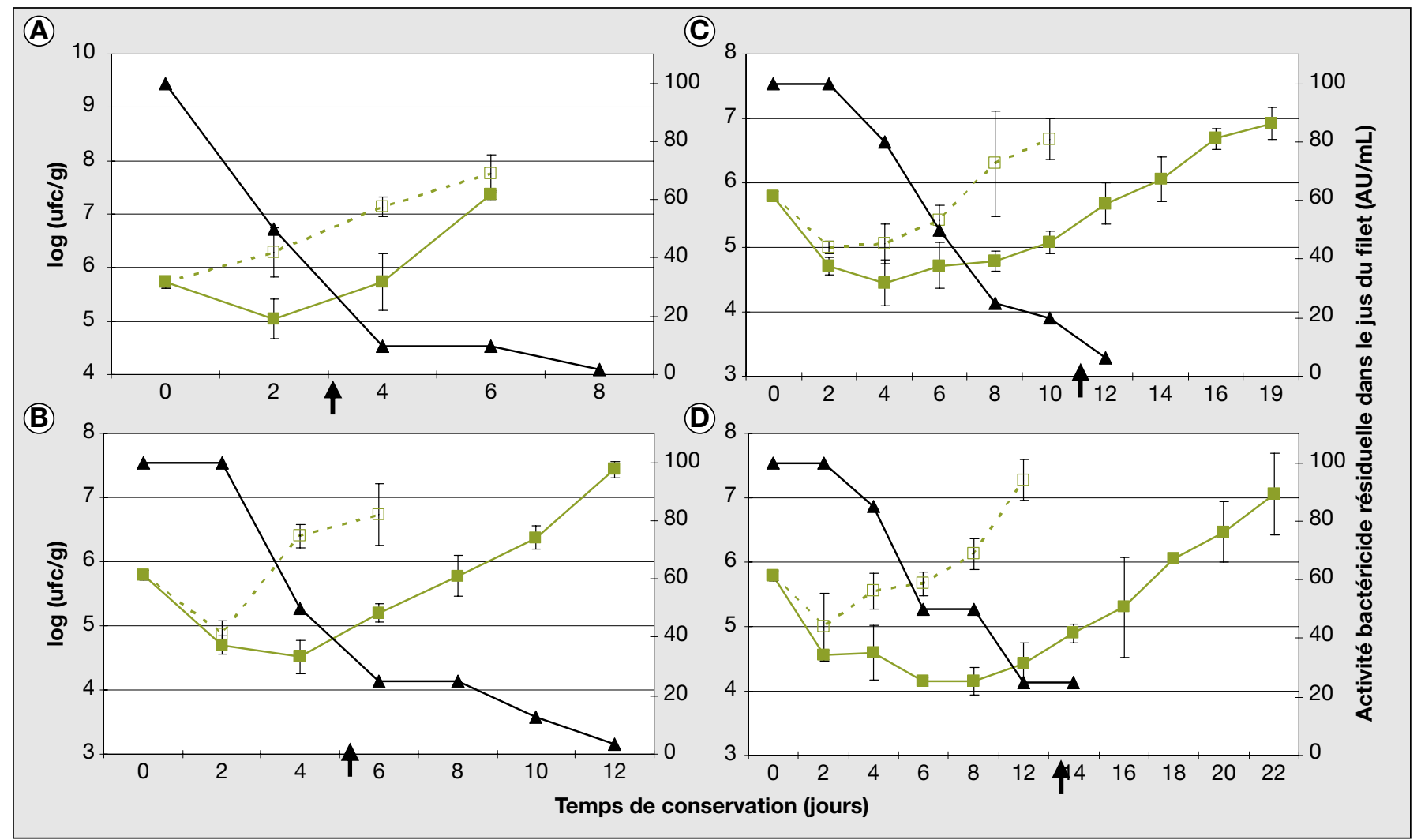

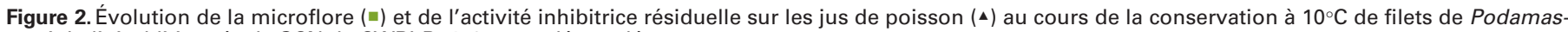
sys jubelini additionnés de SCN de CWBI-B1410 non salé et salé.

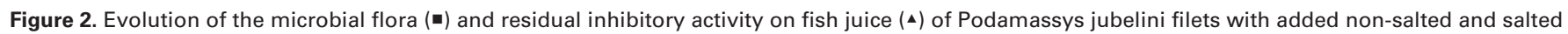
CWBI-B1410 NCS during storage at $10^{\circ} \mathrm{C}$.

La concentration finale en $\mathrm{NaCl}$ dans les jus de poisson atteint $0 \%$ (A), $4 \%$ (B), $6 \%$ (C) et $7 \%$ (D).

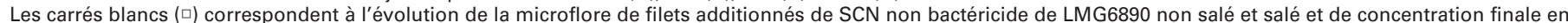
$\mathrm{NaCl}$ atteignant $0 \%(\mathrm{~A}), 4 \%(\mathrm{~B}), 6 \%$ (C) et $7 \%(\mathrm{D})$.

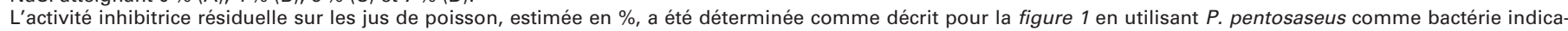
trice et en considérant I'activité mesurée sur le SCN de CWBI-B1410 comme étant de $100 \%$.

Les flèches indiquent le début du phénomène du " rebond ». 


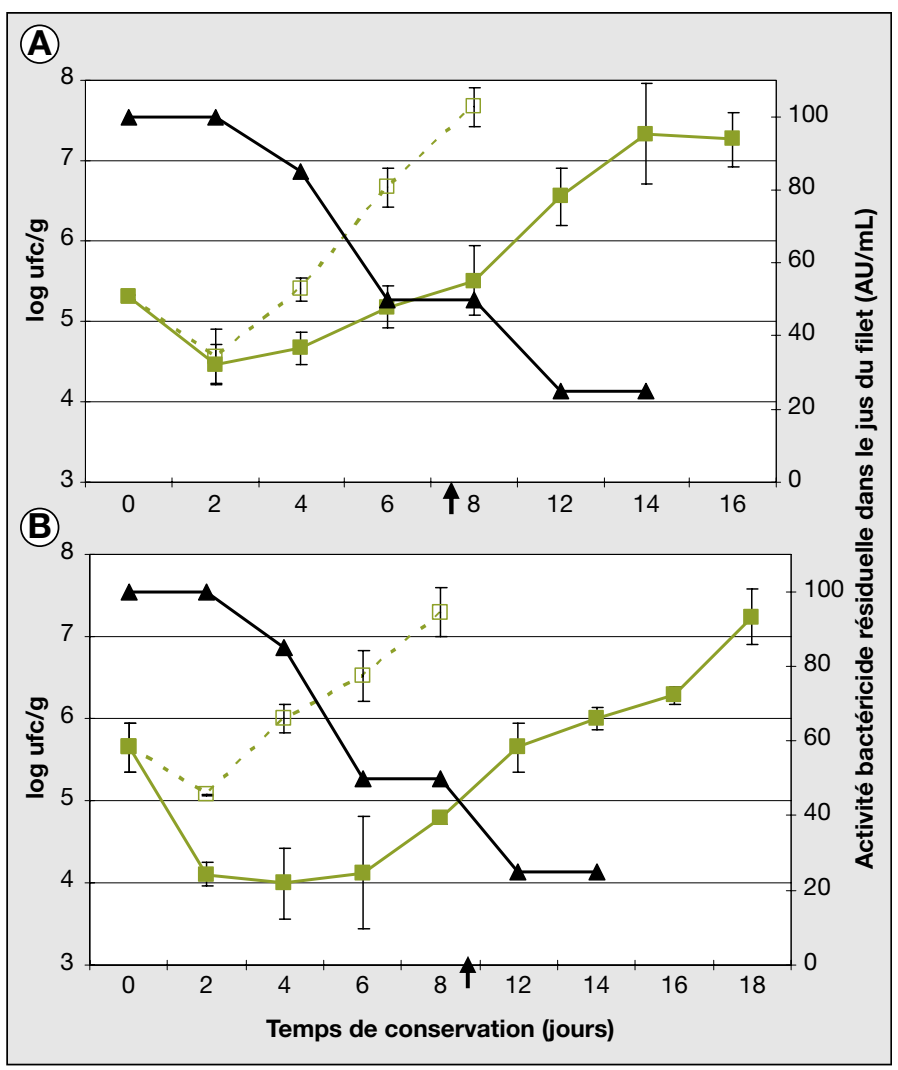

Figure 3. Évolution de la microflore $(\Xi)$ et de l'activité inhibitrice résiduelle sur les jus de poisson ( $\mathbf{\Delta}$ ) au cours de la conservation à $10^{\circ} \mathrm{C}$ de filets de Polydactylis quadrifilis (A) et Arius heudeloti (B) additionnés de SCN de CWBI-B1410 salé (concentration finale en $\mathrm{NaCl}$ atteignant $7 \%$ )

Figure 3. Evolution of the microbial flora ( $\bullet$ ) and residual inhibitory activity on fish juice ( $\Delta)$ of $P o l y d a c-$ tylis quadrifilis $(\mathrm{A})$ and Arius heudeloti $(\mathrm{B})$ with added salted ( $\mathrm{NaCl}$ at final concentration reaching $7 \%$ ) CWBI-B1410 NCS during storage at $10^{\circ} \mathrm{C}$.

Les carrés blancs $(\square)$ correspondent à l'évolution de la microflore de filets additionnés de SCN non bactéricide de LMG6890 salé (concentration finale en $\mathrm{NaCl}$ atteignant $7 \%$ )

L'activité inhibitrice résiduelle sur les jus de poisson, estimée en \%, a été déterminée comme décrit pour la figure 1 en utilisant $P$. pentosaseus comme bactérie indicatrice et en considérant l'activité mesurée sur le SCN de CWBI-B1410 comme étant de $100 \%$.

Les flèches indiquent le début du phénomène du " rebond".

La reprise de la croissance bactérienne à $10{ }^{\circ} \mathrm{C}$ (phénomène de rebond) survient lorsque l'activité antibactérienne résiduelle sur les jus du poisson traité avec le SCN de CWBI-B1410 éventuellement salé est de 25-30 \% (figures 2 et 3). Les bactériocines étant des substances peptidiques, leur dégradation par les enzymes protéolytiques endogènes des poissons explique la baisse progressive de l'activité antibactérienne résiduelle sur le jus des poissons (Goff et al., 1996; Ghalfi et al., 2006).

En plus de son activité antibactérienne, le sel inhibe aussi l'activité des enzymes endogènes. Ceci se produit lorsque la concentration en $\mathrm{NaCl}$ dans la chair du poisson atteint et dépasse 5-6\% (Gérard, 1989), d'où l'amélioration de la stabilité de l'activité inhibitrice résiduelle sur le jus de poissons salés à $7 \%$ (moindre dégradation de la bactériocine), compa- rée à celle sur le jus de poisson moins salé (figure $2 \mathrm{~A}, \mathrm{~B}, \mathrm{C}$ et $\mathrm{D}$ ).

La différence d'efficacité du SCN de CWBI-

B1410 salé à $7 \%$ sur les différents poissons (maigre, moyennement gras et gras) peut être expliquée par une différence d'adsorption ou d'interaction de la bactériocine produite par la souche CWBI-B1410 avec certains composés de la chair des poissons notamment leurs constituants lipidiques (Aasen et al., 2003, Ghalfi et al., 2006, et Zapico et al., 1999).

\section{Conclusion}

Le niveau de la microflore des filets crus de poissons conditionnés artisanalement au Sénégal est à la limite de la salubrité définie à $10^{6}$ ufc/g. L'utilisation de SCN bactéricide de CWBI-B1410 additionné de sel comme conservateur sur les poissons - à concentration finale en $\mathrm{NaCl}$ de $7 \%$-, réduit le niveau de la flore bactérienne de 1,5 log ufc/g et retarde la reprise de sa croissance. Le résultat est une augmentation de la durée de conservation de $12,7,5$ et 8 jours à $10^{\circ} \mathrm{C}$, respectivement pour les filets de poisson maigre, moyennement gras et gras.

Ces résultats suggèrent que cette technologie simple peut constituer un moyen convenable d'améliorer la conservation et la qualité microbiologique des produits halieutiques tropicaux.

\section{Remerciements}

Nous remercions la Coopération universitaire au développement (CUD) de la Belgique qui a financé nos études doctorales réalisées en alternance au Centre wallon de biologie industrielle (CWBI) de la faculté universitaire des sciences agronomiques de Gembloux et au Laboratoire de microbiologie appliquée et génie industriel (L-MAGI) de l'École supérieure polytechnique de l'université Cheikh Anta Diop de Dakar.

\section{Références}

Aasen IM, Markussen S, Moretro T, Katla T, Axellsson L, Naterstad K. Interactions of the bacteriocins sakacin $P$ and nisin with food constituents. Int J of Food Microbiol $2003 ; 87$ : 35-43.

Baird-Parker TC. The production of microbiologically safe and stable foods. In: Lund BM, Baird Parker TC, Gould GW, eds. The Microbiological safety and quality of food. Gaistherburg: Aspen Publishers, Inc. 2000.

Barefoot SF, Klaenhammer TR. Detection and activity of lacticin B, a bacteriocin produced by Lactobacillus acidophilus. Appl Environ Microbiol 1983 ; 45 : 1808-15.

Boyd LC, Green DP, Lepors LA. Quality changes of pond raised hybrid striped bass during chillpack and refrigerated storage. J Food Sci $1992 ; 57: 59-62$

De Vuyst L, Vandamme EJ. Antimicrobial potential of Lactic acid bacteria. In : De Vuyst $\mathrm{L}$, Vandamme EJ, eds. Bacteriocins of lactic acid bacteria: Microbiology, genetics and applications. London: Blackie Academic and Professional, 1994.

Degnan AJ, Kaspar CW, Otwell S, Tamplin ML, Luchansky JB. Evaluation of lactic acid bacterium fermentation products and food grade chemicals to control Listeria monocytogenes in blue crab (Callinectes sapidus) meat. Appl Environ Microbiol 1994 ; 60:3198-203. 
Diei-Ouadi Y. Minced sardinella fillets in fish landing and marketing sites in Senegal. FAO fisheries Circular No 999:FIIU/C999(EN). Rome: FAO, 2005.

Diop $M B$, Tine $E$, Dubois-Dauphin $R$, NGom EHA, Thonart P. Description of plasmidic gene encoding nisin $A$, a bacteriocin from $L$. lactis subsp. lactis CWBI-B1410 isolated from Senegalease traditional fermented millet flour. EMBL/Genbank/DDBJ 2007; Accession number EF371000.

Diop MB, Tine E, NGom EHA, Dubois-Dauphin R, Destain J, Thonart P. Bacteriocin producers from traditional food products. Biotechnol Agron Soc Environ 2007 ; 11 : 1-64.

Direction des pêches maritimes (DOPM). Rapport 2004. Dakar (Sénégal): Direction des pêches maritimes, 2004.

Eïnarsson $H$, Lauzon HL. Biopreservation of brined shrimp (pandalus borealis) by bacterio cins from lactic acid bacteria. Appl Environ Microbiol $1995 ; 61$ : 669-76.

Fellows P. Meat, Fish and dairy products. In Fellows P, ed. Traditional foods processing for Profits. London: Intermediate Technology Publication, 1997.
Folch J, Lees M, Sloane-Stanley G. A simple method for isolation and purification of total lipids from animal tissues. J Biol Chem 1957; 226 : 497-504.

Gérard P. Méthode moderne de séchage du poisson. Nouv Sci Technol 1989; 7 : 127-30.

Ghalfi H, Allaoui A, Destain J, Benkerroum N, Thonart $P$. Bacteriocin activity by Lactobacillus curvatus CWBI-B28 to inactivate Listeria monocytogenes in Cold-smoked salmon during $4^{\circ} \mathrm{C}$ storage. J Food Prot 2006; 59 : 1066-71.

Goff JH, Bhunia AK, Johnson MG. Complete inhibition of low levels of Listeria monocytogenes on refrigerated chicken meat with pediocin $\mathrm{AcH}$ bound to heat-killed $P$. acidilactici cells. J Food Prot 1996 ; 59 : 1187-92.

Gram L, Dalgaard P. Fish spoilage bacteriaproblems and solutions. Cur Opinion Biotechnol $2002 ; 13: 262-6$.

Gram L. Spoilage of three Senegalese fish species stored in ice at ambient temperature. In : Bligh EH, ed. Seafood Science and Technology. Fishing News Books. Oxford: Blackwell, 1992. 1992
Guiraud JP. Microbiologie alimentaire. Paris: Dunod, 1998.

Olsen SJ, Mackinnon LC, Goulding JS, Bean $\mathrm{NH}$, Slutsker L. Surveillance for foodbornedisease outbreaks- United States, 1993-1997. Morbidity Weekly report CDC. Surveill Summ $2000 ; 49: 1-62$.

Oregon State University (OSU) Extension Service. Fish pickling for home use. PNW 183. 1993. http:// extension.oregonstate.edu [consulté en juillet, 2006].

Stansby M. Proximate composition of fish. In : Heen $\mathrm{E}$, Kreuzer $\mathrm{R}$, eds. Fish in nutrition. Oxford: Fishing News (Books) Ltd, 1962.

Wessels $\mathrm{S}$, Huss $\mathrm{HH}$. Suitability of L. lactis subsp. lactis ATCC 11454 as a protective culture for lightly preserved fish products. Food Microbiol 1996 ; 13 : 323-32.

Zapico $\mathrm{P}$, De Paz M, Medina $\mathrm{M}$, Nunĕz, $\mathrm{M}$. The effect of homogenization of whole milk, skim and milk fat on nisin activity against Listeria innocua. Int J Food Microbiol 1999, 46:1517. 